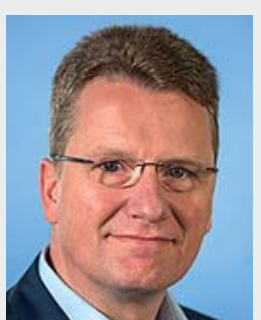

Ulf Culemann

\title{
Vom Schockraum zur Reha
}

\section{Liebe Kolleginnen und Kollegen,}

die Notfallbehandlung schwerverletzter Patienten ist in den letzten Jahren kontinuierlich weiterentwickelt und die medizinische Behandlung immer weiter verbessert und in ihren Abläufen konkretisiert worden. Durch Traumaregisterbeteiligung und Zertifizierung konten wir die Behandlungsqualität stetig vorantreiben und steigern.

Im vorliegenden Themenheft schauen wir ganz gezielt den Behandlungpfad eines polytraumatisierten Patienten an. Hier widmen wir uns einerseits der präklinischen Versorgung des schwerverletzten Patienten inklusive der Aufnahme im Schockraum, aber auch der interdisziplinären Weiterbehandlung in der Klinik. Dass das Zeitmanagement bei der Versorgung eines polytraumatisierten Patienten immer noch einen wichtigen Beitrag zur zielgerichteten Versorgung leistet, wird auch in der Beantwortung der Frage nach konservativer versus operativer Therapie des SHT im Heft verdeutlicht.

Ein selten beachteter, aber mindestens genauso wichtiger Aspekt der Vorbereitung auf den „Ernstfall“ sind die Beiträge zur Materialvorhaltung im Schockraum inklusive der radiologischen Sichtweise, dies dürfte insbesondere auch die Leitenden Pflegekräfte der zentralen Notaufnahmen unserer Kliniken interessieren!

Dass wir derzeit durch Bildung von Katastrophenstäben mit Reduzierung der Kapazitäten auf das Wesentliche genau diese Vorbereitung und Ablaufszenarien umsetzen müssen, ist ein guter Grund, auch für den Bereich der Polytraumaversorgung durch Information und Ablaufverbesserung weiterhin gut gerüstet zu sein. Hierzu soll das vorliegende Heft einen Betrag leisten; ich wünsche Ihnen hierzu viel Vergnügen bei der Lektüre.

Bleiben Sie gesund!

Prof. Dr. med. Ulf Culemann

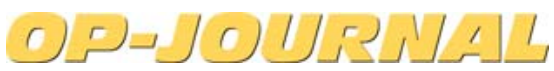

mitbegründet von

Prof. Dr. med. Günther Hierholzer

Prof. Dr. med. h. c. mult. Siegfried Weller

Herausgeber

AOTrauma Deutschland e. V.

Präsident: Prof. Dr. med. Ulrich Stöckle, Berlin
Schriftleiter

Prof. Dr. med. Ulf Culemann, Celle

Prof. Dr. med. Christof Müller, Karlsruhe

Prof. Dr. med. Ulrich Stöckle, Berlin

Beirat

Priv.-Doz. Dr. med. Hermann Josef Bail, Nürnberg

Prof. Dr. med. Karl-Heinz Frosch, Hamburg

Priv.-Doz. Dr. med. dent. Alexander Gröbe,

Hannover
Prof. Dr. med. Marius Johann Baptist Keel, FACS, Bern, Schweiz

Prof. Dr. med. Dr. h. c. Edgar Mayr, Augsburg Prof. Dr. med. Wolfgang Lehmann, Göttingen Prof. Dr. med. Hans-Christoph Pape, Zürich Prim. Dr. med. Michael Plecko, Graz, Österreich Prof. Dr. med. Klaus Schaser, Dresden Alexander Motzny, Tübingen 